\title{
PROPERTIES OF LYUBEZNIK NUMBERS UNDER LOCALIZATION AND POLARIZATION
}

\author{
ARINDAM BANERJEE, LUIS NÚÑEZ-BETANCOURT, AND KOHJI YANAGAWA
}

\begin{abstract}
We exhibit a global bound for the Lyubeznik numbers of a ring of prime characteristic. In addition, we show that for a monomial ideal, the Lyubeznik numbers of the quotient rings of its radical and its polarization are the same. Furthermore, we present examples that show striking behavior of the Lyubeznik numbers under localization. We also show related results for generalizations of the Lyubeznik numbers.
\end{abstract}

\section{INTRODUCTION}

In 1993 Lyubeznik [Lyu93] introduced a family of invariants for a local ring containing a field, $R$, today called Lyubeznik numbers and denoted by $\lambda_{i, j}(R)$ (see Section 2.1). These numbers have been shown to have multiple connections; for instance, they relate to singular and ètale cohomology [BB05, GLS98, Lyu93], to the Hochster-Huneke graph [Lyu06, Zha07], and to projective varieties [Zha11, Swi14]. These connections have motivated multiple generalizations; for instance, for mixed characteristic rings [NBW13], and rings of equal-characteristic from a differential perspective [ÄM04, NBW14].

In this article, we study the behavior of these invariants under localization. The first result obtained in this context is that the Lyubeznik numbers are bounded globally over rings of positive characteristic, which resemble behavior of Bass numbers under localization (see 3.0.1).

Theorem (see Theorem 3.3). Let $R$ be a ring which is a quotient of a regular Noetherian regular ring of finite dimension and positive characteristic $p>0$. Then, there exists a positive integer $B$ such that

$$
\lambda_{i, j}\left(R_{\mathfrak{p}}\right) \leq B
$$

for every $i, j \in \mathbb{N}$ and $\mathfrak{p} \in \operatorname{Spec}(R)$.

The previous claim was proven for algebras finitely generated over a field of characteristic zero or an algebraically closed field of prime characteristic by Puthenpurakal [Put13]. We point out that his result deals only with localization at maximal ideals.

Unfortunately, the Lyubeznik numbers do not behave much better than it is stated in the previous theorem. We show an example of a Stanley-Reisner ring whose highest Lyubeznik number could either decrease or increase under localization (see Example 4.1). In fact, we show a method to build a Stanley-Reisner ring with all but one Lyubeznik number vanishing and with a localization that, surprisingly, have many positive Lyubeznik numbers (see Remark 4.6).

An operation related with localization of Stanley-Reisner rings is polarization (see Remark 2.10). Given a monomial ideal $I$, not necessarily radical, in a polynomial ring $S$, we consider the polarization ideal $\widetilde{I}$ in the polarization ring $\widetilde{S}$. We compare the Lyubeznik number at the maximal homogeneous ideal of $S / \sqrt{I}$ and $\widetilde{S} / \widetilde{I}$. 
Theorem (see Theorem 5.1). Let $S=K\left[x_{1}, \ldots, x_{n}\right]$ be a polynomial ring, $\mathfrak{m}=\left(x_{1}, \ldots, x_{n}\right)$ and $I \subset S$ be a monomial ring. Let $\widetilde{I}$ denote the polarization of $I$, and $\widetilde{S}=K\left[x_{r, s}\right]$ denote the polarization ring. Let $\mathfrak{n}=\left(x_{i, j}\right)$, and $h=\operatorname{dim}(\widetilde{S} / \widetilde{I})-\operatorname{dim}(S / I)$. Then,

$$
\lambda_{i-h, j-h}\left(S_{\mathfrak{m}} / I S_{\mathfrak{m}}\right)=\lambda_{i, j}\left(\widetilde{S}_{\mathfrak{n}} / \widetilde{I}_{\mathfrak{n}}\right)
$$

for every $i, j \in \mathbb{N}$.

This result gives surprisingly different behavior of Lyubeznik numbers under localization and polarization. We also study the behavior of the generalized Lyubeznik numbers, $\lambda_{0}^{i}(R)$, for rings of equal characteristic under localization and polarization for Stanley-Reisner rings (see Propositions 4.7 and 5.4). As a consequence of our methods, we observe similar behavior for finer invariants given by the multiplicities of characteristics cycles.

\section{BACKGROUND}

2.1. Local cohomology and Lyubeznik numbers. In this section we recall definitions and properties of local cohomology and Lyubeznik numbers that we discuss in further sections. We refer the interested reader to [BS98, ILL $\left.{ }^{+} 07\right]$ for local cohomology and [NBWZ14] for Lyubeznik numbers.

Let $R$ be a Noetherian ring, $I \subset R$ an ideal, and $M$ an $R$-module. Suppose that $I$ is generated by $\underline{f}=f_{1}, \ldots, f_{\ell}$. We consider the Čech complex:

$$
C^{\bullet}(f ; M): 0 \rightarrow M \rightarrow \oplus_{j} M_{f_{j}} \rightarrow \oplus_{i<j} M_{f_{i} f_{j}} \rightarrow \ldots \rightarrow M_{f_{1} \cdots f_{\ell}} \rightarrow 0
$$

The $j$-th cohomology of this complex, $H_{I}^{j}(M)$, is called the local cohomology module supported at the ideal $I=\left(f_{1}, \cdots, f_{\ell}\right)$. We point out that $H_{I}^{j}(M)$ does not depend on the choice of generators for $I$. We note that local cohomology commutes with flat extensions; in particular, $\left(H_{I}^{i}(R)\right)_{\mathfrak{p}}=H_{I}^{i}\left(R_{\mathfrak{p}}\right)$ for every prime ideal $\mathfrak{p} \subset R$.

Definition 2.1. Let $M$ be an $R$-module and $\mathfrak{p}$ be a prime ideal. We define the $i$-th Bass number of $M$ with respect to $\mathfrak{p}$, denoted $\mu^{i}(\mathfrak{p}, M)$, as $\operatorname{dim}_{R_{\mathfrak{p}} / \mathfrak{p} R_{\mathfrak{p}}} \operatorname{Ext}_{R}^{i}\left(R_{\mathfrak{p}} / \mathfrak{p} R_{\mathfrak{p}}, M_{\mathfrak{p}}\right)$.

Suppose that $M$ is an $R$-module. Let $0 \rightarrow M \rightarrow E^{0} \rightarrow E^{1} \rightarrow \ldots$ be a minimal injective resolution for $M$. The number of copies of the injective hull of $R / \mathfrak{p}, E_{R}(R / \mathfrak{p})$, in $E^{i}$ is given by $\mu^{i}(\mathfrak{p}, M)$.

Theorem 2.2 (see [HS93, Theorem 2.1] and [Lyu93, Theorem 3.4]). Let $S$ be a regular ring containing a field. Every local cohomology module $H_{I}^{i}(S)$ has finite Bass numbers. That is, for every prime ideal $\mathfrak{p} \subset S, \operatorname{dim}_{\operatorname{Ext}_{S_{\mathfrak{p}}}^{i}}\left(S_{\mathfrak{p}} / \mathfrak{p} S_{\mathfrak{p}}, H_{I}^{j}\left(S_{\mathfrak{p}}\right)\right)$ is finite.

Definition 2.3 (see [Lyu93, Theorem-Definition 4.1]). Let $(R, \mathfrak{m}, K)$ be a local ring containing a field. By the Cohen Structure Theorems, there exists a surjective homomorphism $\pi: S \rightarrow \widehat{R}$, where $\widehat{R}$ is the completion of $R$, and $S=K\left[\left[x_{1}, \ldots, x_{n}\right]\right]$ for some $n$. If $I=\operatorname{Ker}(\pi)$, the Lyubeznik number of $R$ with respect to integers $i, j \geq 0$ is defined as

$$
\lambda_{i, j}(R)=\mu^{i}\left(\mathfrak{m}, H_{I}^{n-j}(S)\right)=\operatorname{dim}_{K} \operatorname{Ext}_{S}^{i}\left(K, H_{I}^{n-j}(S)\right),
$$

which is finite and only depends on the ring $R$ and the integers $i$ and $j$. If $d=\operatorname{dim}(R)$, $\lambda_{d, d}(R)$ is called the highest Lyubeznik number of $R$ (this is justified because $\lambda_{i, j}(R)=0$ if $i>d$ or $j>d)$. We can arrange the Lyubeznik number in a matrix $\left(\lambda_{i, j}(R)\right)$. We say that $R$ has a trivial Lyubeznik table if $\lambda_{d, d}(R)=1$ and all the other vanish. 
Definition 2.4 ([HH94]). Let $R$ be a local ring. The Hochster-Huneke graph $\Gamma_{R}$ of $R$ is defined as follows. Its vertices are the minimal prime ideals, $\mathfrak{p}$, of $R$ such that $\operatorname{dim}(R)=$ $\operatorname{dim}(R / \mathfrak{p})$. Two different vertices $P$ and $\mathfrak{q}$ are joined by an edge if and only if $\mathrm{ht}_{R}(\mathfrak{p}+\mathfrak{q})=1$.

Theorem 2.5 (see [Zha07, Main Theorem]). Let $R$ be a $d$-dimensional local ring that contains a field. Then $\lambda_{d, d}(R)$ is equal to the number of connected components of the Hochster-Huneke graph of $B$, where $B=\widehat{R^{s h}}$ is the completion of the strict Henzelization of $R$.

Remark 2.6. The set of associated primes of a squarefree monomial ideal depends only on the minimal monomial generators (cf. [HH11, Proposition 1.2.2 and Corollary 1.3.10]). Therefore, the Hochster-Huneke graph for a Stanley-Reisner ring is independent of the base field.

2.2. Monomial ideals and polarization. In Sections 4 and 5, we study the behavior of Lyubeznik numbers of Stanley-Reisner rings. For more detail about local cohomology with support in monomial ideals, we refer to [ĀMGLZA03, ÀM04, ÀMV14, Mus00, Ter99, Yan00, Yan01]. We also refer to [Pee11, Far06, Yan12] for details about polarization.

Definition 2.7. Let $S=K\left[x_{1}, \ldots, x_{n}\right]$. Let $M=\bigoplus_{\alpha \in \mathbb{Z}^{n}} M_{\alpha}$ be finitely generated $\mathbb{Z}^{n}$-graded $S$-module. Given $\beta \in \mathbb{Z}^{n}$, we take the shifted module $M(\beta)$ by $(M(\beta))_{\alpha}=M_{\alpha+\beta}$.

We recall the category of squerefree modules.

Definition 2.8 ([Yan00]). Let $S=K\left[x_{1}, \ldots, x_{n}\right]$. We say a finitely generated $\mathbb{N}^{n}$-graded $S$-module $M=\bigoplus_{\alpha \in \mathbb{N}^{n}} M_{\alpha}$ is squarefree, if the multiplication maps $M_{\alpha} \ni y \longmapsto x_{i} y \in M_{\alpha+\mathbf{e}_{i}}$ is bijective for all $\alpha \in \mathbb{N}^{n}$ and all $i \in \operatorname{supp}(\alpha):=\left\{i \mid \alpha_{i} \neq 0\right\}$.

Here we list some basic properties of these graded modules.

- For a monomial ideal $I$, it is a squarefree $S$-module, if and only if $S / I$ is a squarefree module, if and only if $I=\sqrt{I}$. The free modules $S$ itself and the $\mathbb{Z}^{n}$-graded canonical module $\omega_{S}=S(\mathbf{- 1})$ are squarefree. Here $\mathbf{1}=(1,1, \ldots, 1) \in \mathbb{N}^{n}$.

- Let ${ }^{*} \bmod S$ be the category of $\mathbb{Z}^{n}$-graded finitely generated $S$-modules, and Sq $S$ its full subcategory consisting of squarefree modules. Then $\mathrm{Sq} S$ is an Abelian subcategory of $* \bmod S$.

For each $\alpha \in \mathbb{N}^{n}$, E. Miller [Mil00] introduced the notion of positively $\alpha$-determined $S$ modules. Here we do not gives the precise definition, but just list some basic properties.

- A monomial ideal $I$ (equivalently $S / I$ ) is positively $\alpha$-determined if and only if $I$ is generated by monomials of the form $x^{\beta}$ for some $\beta \preceq \alpha$.

- A positively 1-determined $S$-module is nothing other than a squarefree $S$-module.

- Let $* \bmod _{\alpha} S$ be the full subcategory of $* \bmod S$ consisting of positively $\alpha$-determined $S$-modules. Then ${ }^{*} \bmod _{\alpha} S$ is an Abelian subcategory of ${ }^{*} \bmod S$.

- If $M \in{ }^{*} \bmod _{\alpha} S$, then $\operatorname{Ext}_{S}^{i}(M, S(-\alpha)) \in{ }^{*} \bmod _{\alpha} S$.

We now fix notation and relate squarefree monomial ideals with simplicial complexes. Set $[n]:=\{1, \ldots, n\}$ and $S=K\left[x_{1}, \ldots, x_{n}\right]$. For a simplicial complex $\Delta \subset 2^{[n]}$, consider a squarefree monomial ideal $I_{\Delta}:=\left(\prod_{i \in \sigma} x_{i} \mid \sigma \subset[n], \sigma \notin \Delta\right)$ of $S$. Any squarefree monomial ideal is of the form $I_{\Delta}$ for some $\Delta$, and $K[\Delta]:=S / I_{\Delta}$ is the Stanley-Reisner ring of $\Delta$ over 
$K$. Let $\mathfrak{m}=\left(x_{1}, \ldots, x_{n}\right)$. The third author [Yan01, Theorem 1.1] showed that the Lyubeznik numbers of $K[\Delta]_{\mathfrak{m}}$ can be obtained from its graded structure:

$$
\lambda_{i, j}\left(K[\Delta]_{\mathfrak{m}}\right)=\operatorname{dim}_{K}\left[\operatorname{Ext}_{S}^{n-i}\left(\operatorname{Ext}_{S}^{n-j}\left(K[\Delta], \omega_{S}\right), \omega_{S}\right)\right]_{0} .
$$

For $\sigma \subset[n]$, let $\mathfrak{p}_{\sigma}:=\left(x_{i} \mid i \notin \sigma\right)$ be a monomial prime ideal of $S$, and set $K[\sigma]:=S / \mathfrak{p}_{\sigma} \cong$ $K\left[x_{i} \mid i \in \sigma\right]$. We remark that $K[\sigma]$ can be seen as a subring of $S$ in the natural way. Recall that the link of $\sigma$ in a simplicial complex $\Delta$ is defined by

$$
\operatorname{lk}_{\Delta} \sigma:=\{\tau \subset[n] \mid \tau \cap \sigma=\emptyset, \tau \cup \sigma \in \Delta\}
$$

It is a simplicial complex again, and we have

$$
K[\Delta]_{\mathfrak{p}_{\sigma}} \cong L\left[\mathrm{lk}_{\Delta} \sigma^{\mathrm{c}}\right]_{\mathfrak{n}},
$$

where $L$ is an extended field of $K, \mathfrak{n}$ is the graded maximal ideal of $L[\sigma]=L\left[x_{i} \mid i \in \sigma\right]$, and we set $\sigma^{\mathrm{c}}:=[n] \backslash \sigma$. From the previous discussion and Equation 2.2.1, we have

$$
\begin{aligned}
\lambda_{i, j}\left(K[\Delta]_{\mathfrak{p}_{\sigma}}\right) & =\lambda_{i, j}\left(L\left[\mathrm{lk}_{\Delta} \sigma^{\mathrm{c}}\right]_{\mathfrak{n}}\right) \\
& =\operatorname{dim}_{L} \operatorname{Ext}_{L[\sigma]}^{\# \sigma-i}\left(\operatorname{Ext}_{L[\sigma]}^{\# \sigma-j}\left(L\left[\mathrm{lk}_{\Delta} \sigma^{\mathrm{c}}\right], \omega_{L[\sigma]}\right), \omega_{L[\sigma]}\right) \\
& =\operatorname{dim}_{K} \operatorname{Ext}_{K[\sigma]}^{\# \sigma-i}\left(\operatorname{Ext}_{K[\sigma]}^{\# \sigma-j}\left(K\left[\mathrm{lk}_{\Delta} \sigma^{\mathrm{c}}\right], \omega_{K[\sigma]}\right), \omega_{K[\sigma]}\right)
\end{aligned}
$$

To control the last term of (2.2.2), we have to step into the theory squarefree modules. We say $\alpha \in \mathbb{N}^{n}$ is squarefree if $\alpha_{i}=0,1$ for all $i$. When $\alpha$ is squarefree, we freely identify $\alpha$ with $\operatorname{supp}(\alpha)$. For example, if $M$ is a $\mathbb{Z}^{n}$-graded module and $\sigma \subset[n], M(\sigma)$ denotes the shifted module of $M$ by the corresponding 0-1 vector. Similarly, $M_{\sigma}$ is the homogeneous component of $M$ with the corresponding degree. If $M$ is a $\mathbb{Z}^{n}$-graded $S$-module, through the ring homomorphism $K[\sigma] \hookrightarrow S$, we can regard

$$
\left.M\right|_{\sigma}:=\bigoplus_{\substack{\alpha \in \mathbb{N}^{n} \\ \operatorname{supp}(\alpha) \subset \sigma}} M_{\sigma}
$$

as a $\mathbb{N}^{\# \sigma}$-graded $K[\sigma]$-module. For a squarefree $S$-module $M$, it is easy to see that

$$
\mathfrak{l}_{\sigma}(M):=\left.M\left(\sigma^{\mathrm{c}}\right)\right|_{\sigma}
$$

is a squarefree $K[\sigma]$-module with

$$
\left(\mathfrak{l}_{\sigma}(M)\right)_{\tau}=M_{\sigma^{\mathrm{c}} \cup \tau}
$$

for each $\tau \subset \sigma$. Hence we have an exact functor $\mathfrak{l}_{\sigma}: \operatorname{Sq} S \rightarrow \operatorname{Sq} K[\sigma]$. Straightforward computation shown that

$$
\mathfrak{l}_{\sigma}(K[\Delta]) \cong K\left[\mathrm{lk}_{\Delta} \sigma^{\mathrm{c}}\right]=K[\sigma] / I_{\mathrm{lk}_{\Delta} \sigma^{\mathrm{c}}}
$$

In particular, for $\tau \subset[n]$, we have

$$
\mathfrak{l}_{\sigma}(K[\tau]) \cong \begin{cases}K[\tau \cap \sigma] & \text { if } \tau \supset \sigma^{\mathrm{c}} \\ 0 & \text { otherwise }\end{cases}
$$


Definition 2.9. Let $g=x_{1}^{\alpha_{1}} \cdots x_{n}^{\alpha_{n}}$ be a monomial in $S=K\left[x_{1}, \ldots, x_{n}\right]$. We define another monomial $g^{\prime}$ in the polynomial ring $\widetilde{S}=K\left[x_{i, j}\right]$ where

$$
g^{\prime}=\prod_{i=1}^{n} x_{i, 1} x_{i, 2} \cdots x_{i, \alpha_{i}} .
$$

If $I=\left(g_{1}, \ldots, g_{k}\right)$ is an ideal in $S$, the ideal $\widetilde{I}=\left(g_{1}^{\prime}, \ldots, g_{k}^{\prime}\right)$ in $\widetilde{S}$ is called the polarization of $I$.

Polarization is a classical technique constructing a squarefree monomial ideal $\widetilde{I} \subset \widetilde{S}$ from a general monomial ideal $I \subset S$. There are relations between the singularity of a monomial ideal and its polarization. For instance, the Betti numbers of $S / I$ over $S$ and $\widetilde{S} / \widetilde{I}$ over $\widetilde{S}$ are same (cf. [Pee11, Theorem 21.10]). As a consequence, $S / I$ is Cohen-Macaulay if and only if $\widetilde{S} / \widetilde{I}$ is Cohen-Macaulay.

In [Yan12], the third author extended the polarization operation $I \mapsto \widetilde{I}$ to the functor $\operatorname{pol}_{\alpha}:{ }^{*} \bmod { }_{\alpha} S \rightarrow$ Sq $\widetilde{S}$. Here $\operatorname{pol}_{\alpha}(I)=\widetilde{I}$ and $\operatorname{pol}_{\alpha}(S / I)=\widetilde{S} / \widetilde{I}$. Moreover,

$$
\Theta:=\left\{x_{i, 1}-x_{i, j} \mid 1 \leq i \leq n, 2 \leq j \leq a_{i}\right\} \subset \widetilde{R}
$$

forms a $\operatorname{pol}_{\alpha}(M)$-regular sequence, and gives an isomorphism $\operatorname{pol}_{\alpha}(M) \otimes_{\widetilde{S}} \widetilde{S} /(\Theta) \cong M$. ${ }^{1}$ Here we just remark that $\left[\mathrm{pol}_{\alpha}(M)\right]_{\mathbf{0}} \cong M_{\mathbf{0}}$. The "reversed copy" $\mathrm{pol}^{\alpha}$ of pol $\alpha$ is also a polarization functor ${ }^{*} \bmod _{\alpha} S \rightarrow \mathrm{Sq} \widetilde{S}$, but use the convention that $\mathrm{pol}^{\alpha}\left(x^{\beta}\right)=\prod x_{i, \alpha_{i}} x_{i, \alpha_{i}-1} \cdots x_{i, \alpha_{i}-\beta_{i}+1} \in$ $\widetilde{S}$.

We now present a well-known relation between polarization and localization, which will play a major role in the proof of Proposition 5.4.

Remark 2.10. Suppose that $I$ is a monomial ideal in a polynomial $\operatorname{ring} S=K\left[x_{1}, \ldots, x_{n}\right]$, and $\widetilde{I}$ is its polarization in $\widetilde{S}=K\left[x_{i, j}\right]$. We consider an inclusion $\iota: S \rightarrow \widetilde{S}$ given by $x_{i} \mapsto x_{i, 1}$. Let $\mathfrak{p}=\left(x_{i, 1}, \ldots, x_{n, 1}\right)$ be the prime ideal generated by the image of the variables $x_{i}$. We have that

$$
\left(\frac{\widetilde{S}}{\widetilde{I}}\right)_{\mathfrak{p}}=\left(\frac{\widetilde{S}}{\iota(\sqrt{I}) \widetilde{S}}\right)_{\mathfrak{p}}
$$

Moreover, if $L=\operatorname{Frac}\left(K\left[x_{i, j}\right]_{j>1}\right)$, and $T=L\left[\left[x_{1}, \ldots, x_{n}\right]\right]$

$$
\widehat{(\widetilde{S} / \widetilde{I})_{\mathfrak{p}}} \cong \frac{T}{\sqrt{I} T}
$$

2.3. F-modules. We recall some definitions and properties of the theory of $F$-modules introduced by Lyubeznik [Lyu97].

A morphism of rings $\varphi: R \rightarrow S$ gives a change of base functor, $S \otimes_{R} M$, from the category of $R$-modules to $S$-modules. Suppose that $S$ is a regular ring of prime characteristic $p>0$. We are interested in the case when $S=R$ and $\varphi$ is the Frobenius morphism, and the functor is denoted by $\mathcal{F} M$ [PS73]. For example, if $M$ is the cokernel of a matrix $\left(a_{i, j}\right)$, then $\mathcal{F} M$ is the cokernel of $\left(a_{i, j}^{p}\right)$.

\footnotetext{
${ }^{1}$ An essentially same functor had been introduced by Sbarra [Sba01], but we use the convention of [Yan12]
} here. 
Definition 2.11 (see [Lyu97, Definition 1.1]). Let $S$ be a regular ring of prime characteristic $p>0$. An $S$-module, $\mathcal{M}$, is an $F$-module if there exists an isomorphism of $S$-modules $\nu: \mathcal{M} \rightarrow F \mathcal{M}$.

If $M$ is an $S$-module and $\alpha: M \rightarrow \mathcal{F} M$ is a morphism of $S$-modules, we consider

$$
\mathcal{M}=\lim _{\rightarrow}\left(M \stackrel{\alpha}{\rightarrow} \mathcal{F} M \stackrel{\mathcal{F} \alpha}{\rightarrow} \mathcal{F}^{2} M \stackrel{\mathcal{F}^{2} \alpha}{\rightarrow} \ldots\right)
$$

We have that $\mathcal{M}$ is an $F$-module with $\mathcal{M} \stackrel{\alpha}{\rightarrow} \mathcal{F} \mathcal{M}$ as structure isomorphism, if $M$ is a finitely generated $S$-module, we say that $\mathcal{M}$ is an $F$-finite $F$-module with generator $\alpha: M \rightarrow \mathcal{F} M$.

Remark 2.12. Let $S$ be a regular ring of prime characteristic $p>0$. Let $I$ and $J$ be ideals of $S$. Then $H_{I}^{i}(S)$ and $H_{I}^{i} H_{J}^{j}(S)$ are $F$-finite $F$-modules.

Theorem 2.13 (see [Lyu97, Theorem 2.11]). Let $S$ be a regular ring of prime characteristic. Every $F$-finite $F$-module, $M$, has finite Bass numbers. That is, for every prime ideal $\mathfrak{p} \subset S$, $\operatorname{dim} \operatorname{Ext}_{S_{\mathfrak{p}}}^{i}\left(S_{\mathfrak{p}} / \mathfrak{p} S_{\mathfrak{p}}, M_{\mathfrak{p}}\right)$ is finite.

2.4. $D$-modules. In this section, we recall some relevant facts about $D$-modules. We refer the interested reader to [Bjö79, Bjö72, Cou95].

Definition 2.14. Suppose that $S$ is either $K\left[x_{1}, \ldots, x_{n}\right]$ or $K\left[\left[x_{1}, \ldots, x_{n}\right]\right]$. We define the rings of $K$-linear differential operators of $S$ by

$$
D_{K}(S)=S\left\langle\frac{1}{t !} \frac{\partial}{\partial x_{i}} \mid t \in \mathbb{N}, 1 \leq i \leq n\right\rangle \subseteq \operatorname{Hom}_{K}(S, S) .
$$

We have that $S_{f}$ is an $D_{K}(S)$-module of finite length for every $f \in S$. As a consequence, the local cohomology modules $H_{I}^{j}(S)$ and $H_{\mathfrak{m}}^{i} H_{I}^{j}(S)$ have finite length as $D_{K}(S)$-modules [Lyu00, Corollary 6].

Definition 2.15 ([NBW14, Definition 4.3]). Let $(R, \mathfrak{m}, K)$ be a local ring containing a field and let $\widehat{R}$ be its completion at $\mathfrak{m}$. For $L$ a coefficient field of $\widehat{R}$, by Cohen's Structure Theorems there exists a surjection $\pi: S \rightarrow \widehat{R}$, where $S=K\left[\left[x_{1}, \ldots, x_{n}\right]\right]$ for some $n \geq 1$, and such that $\pi(K)=L$. The $i$-th generalized Lyubeznik number of $R$ with respect to $L$ is defined as

$$
\lambda_{0}^{i}(R ; L):=\operatorname{length}_{D_{K}(S)} H_{I}^{n-i}(S)
$$

This number is finite and depends only on $R$ and $L$.

In the previous definition, we considered very specific generalized Lyubeznik numbers. The definition is more general and includes the original Lyubeznik numbers.

Remark 2.16. If in the definition above, $R$ is a localization at the homogeneous maximal ideal of an Stanley-Reisner ring, we have the generalized Lyubeznik numbers could depend on the characteristic of the coefficient field, but not on the choice of a specific field. This is because one can relate the length of $H_{I}^{n-i}(S)$ with length as a straight module (cf. [Yan01, Proposition 2.10 and Remark 2.12] and [NBW14, Theorem 6.6]).

Remark 2.17. If $S=K\left[\left[x_{1}, \ldots, x_{n}\right]\right]$ is a power series and $I$ is a monomial ideal, then there exists a filtration

$$
0=M_{1} \subset M_{2} \subset \ldots \subset M_{\ell}=H_{I}^{i}(S)
$$

such that $M_{j+1} / M_{j} \cong H_{\mathfrak{p}_{\alpha}}^{|\alpha|}(S)$, where $\alpha=\left(\alpha_{1}, \ldots, \alpha_{n}\right) \in\{0,1\}^{n},|\alpha|=\alpha_{1}+\ldots+\alpha_{n}$, and $\mathfrak{p}_{\alpha}=$ $\left(x_{\alpha_{k}} \mid \alpha_{k} \neq 0\right)$. Since $H_{\mathfrak{p}_{\alpha}}^{|\alpha|}(S)$ is a simple $D_{K}(S)$-modules, we have that $\operatorname{length}_{D_{K}(S)} H_{I}^{i}(S)=\ell$. 
Definition 2.18 (see [ĀMGLZA03, Section 2] [ĀM04, Section 3]). Under the notation of Remark 2.17, we take the multiplicities

$$
m_{i,-\alpha}=\#\left\{j \mid M_{j+1} / M_{j} \cong H_{\mathfrak{p}_{\alpha}}^{|\alpha|}(S)\right\}
$$

We define the numbers

$$
\gamma_{i, j}=\sum_{|\alpha|=n-i} m_{j,-\alpha}
$$

which are invariants depending only on $R=S / I$.

Remark 2.19. Under the notation in Remark 2.17 and assuming that $I$ is squarefree, we note that in [AMGLZA03, Proposition 2.1] it is shown that

$$
m_{i, \alpha}=\operatorname{dim}_{K}\left(H_{I}^{i}(S)\right)_{\alpha},
$$

where $\left(H_{I}^{i}(S)\right)_{\alpha}$ denotes the $K$-vector space of degree $\alpha$ in the $\mathbb{Z}^{n}$-graded module $H_{I}^{i}(S)$.

Remark 2.20. Under the notation in Remark 2.17 and assuming that $I$ is squarefree, we note that in [AMGLZA03, Corollary 2.2] it is shown that

$$
m_{i-|\alpha|, \alpha}=\beta_{i,-\alpha}\left(I^{\vee}\right)
$$

where $\beta_{i,-\alpha}\left(I^{\vee}\right)$ denotes the $\alpha$-Betti number of the $\mathbb{Z}^{n}$-graded minimal free resolution of the Alexander dual of $I$ (see also [Mus00, Theorem 3.3]). As a consequence,

$$
\gamma_{i, j}=\sum_{|\alpha|=n-i} m_{j, \alpha}=\sum_{|\alpha|=n-i} \beta_{j+|\alpha|,-\alpha}\left(I^{\vee}\right),
$$

which is the $(i+n-i)$-Betti number of $I^{\vee}$. In addition,

$$
\lambda_{i}^{0}(S / I)=\sum_{\alpha} m_{n-i, \alpha}=\sum_{\alpha} \beta_{n-i+|\alpha|,-\alpha}\left(I^{\vee}\right),
$$

which is the total Betti number of the $(n-i)$-linear strand of the free resolution of $I^{\vee}$.

\section{A GLOBAL BOUND FOR LyUBEZNIK NUMBERS}

In this section we prove first main theorem using $F$-modules theory.

Remark 3.1. Suppose that $(S, \mathfrak{m}, K)$ is a regular local ring. Let $n=\operatorname{dim}(S)$. Let $M$ be a finitely generated $S$-module. Let $\underline{x}=x_{1}, \ldots, x_{n} \in \mathfrak{m}$ be a regular system of parameters, and let $\mathcal{K}(\underline{x},-)$ denote the associated Koszul complex. We have that

$$
\operatorname{Tor}_{i}^{S}(K, M) \cong H_{i}(\mathcal{K}(\underline{x}, M)) \cong H^{n-i}(\mathcal{K}(\underline{x}, M))=\operatorname{Ext}_{S}^{n-i}(K, M) .
$$

Then,

$$
\operatorname{dim}_{K} \operatorname{Tor}_{i}^{S}(K, M)=\operatorname{dim}_{K} \operatorname{Ext}_{S}^{n-i}(K, M) .
$$

Let $S$ denote a regular Noetherian ring, and $M$ be a finitely generated $S$ module. Let $\mathfrak{p}$ be a prime ideal of $S$ and $d_{\mathfrak{p}} \operatorname{denote} \operatorname{dim}\left(S_{\mathfrak{p}}\right)$. Let $\beta_{i}(\mathfrak{p}, M)=\operatorname{dim}_{S_{\mathfrak{p}} / \mathfrak{p} S_{\mathfrak{p}}} \operatorname{Tor}_{i}^{S_{\mathfrak{p}}}\left(S_{\mathfrak{p}} / \mathfrak{p} S_{\mathfrak{p}}, M_{\mathfrak{p}}\right)$. Remark 3.1 has two implications. First, there exists a bound, $B$, given by the maximum rank of the modules appearing in a free resolution for $M$ such that

$$
\mu^{i}(\mathfrak{p}, M)=\beta_{d_{\mathfrak{p}}-i}\left(S_{\mathfrak{p}}, M_{\mathfrak{p}}\right) \leq B
$$

Second, if $\mathfrak{p}^{\prime}$ is another prime ideal contained in $\mathfrak{p}$, we have that

$$
\mu^{i}\left(\mathfrak{p}^{\prime}, M\right)=\beta_{d_{\mathfrak{p}^{\prime}}-i}\left(\mathfrak{p}^{\prime}, M\right) \leq \beta_{d_{\mathfrak{p}^{\prime}}-i}(\mathfrak{p}, M)=\mu^{d_{\mathfrak{p}}-d_{\mathfrak{p}^{\prime}}+i}(\mathfrak{p}, M) .
$$


We need to recall a basic property of $F$-finite $F$-modules.

Lemma 3.2 (see [Lyu97, Remark 2.13]). Suppose that $(S, \mathfrak{m}, K)$ is a regular local ring of positive characteristic. Let $\mathcal{M}$ be an $F$-module with a generator $M \stackrel{\alpha}{\rightarrow} \mathcal{F} M$. Then,

$$
\mu^{i}(K, \mathcal{M}) \leq \mu^{i}(K, M)
$$

Theorem 3.3. Let $S$ be a regular Noetherian ring of prime characteristic $p$ and dimension $n$. Let $\mathcal{M}$ be an $F$-finite $F$-module. Then, there exists an integer $B$ such that

$$
\mu^{i}(\mathfrak{p}, \mathcal{M}) \leq B
$$

for every $\mathfrak{p} \in \operatorname{Spec}(S)$, and $i \in \mathbb{N}$. In particular, for every ideal $I \subset S$, the Lyubeznik numbers of $R=S / I, \lambda_{i, j}\left(R_{\mathfrak{p}}\right)=\mu^{i}\left(\mathfrak{p}, H_{I}^{n-j}(S)\right)$, are bounded.

Proof. Let $n=\operatorname{dim} S$. Let $M \rightarrow \mathcal{F} M$ be a generator for $\mathcal{M}$ such that $M$ is a finitely generated $R$-module. There exists a free resolution for $M$

$$
G_{\bullet}: \ldots \rightarrow G_{i} \rightarrow \ldots \rightarrow G_{0} \rightarrow M \rightarrow 0
$$

such that $b_{i}=\operatorname{rank}\left(G_{i}\right)$ is finite. Let

$$
B=\max \left\{b_{i} \mid i=1, \ldots, n\right\} .
$$

By the characterization of Betti numbers as rank of the terms in a minimal free resolution, we have that

$$
\operatorname{dim}_{S_{\mathfrak{p}} / \mathfrak{p} S_{\mathfrak{p}}}\left(\operatorname{Tor}_{i}^{S_{\mathfrak{p}}}\left(S_{\mathfrak{p}} / \mathfrak{p} S_{\mathfrak{p}}, M_{\mathfrak{p}}\right)\right) \leq b_{i}
$$

for every $i \in \mathbb{N}$, and prime ideal $\mathfrak{p} \subset S$. We have that $M_{\mathfrak{p}} \rightarrow \mathcal{F}_{S_{\mathfrak{p}}} M_{\mathfrak{p}} \cong(\mathcal{F} M)_{\mathfrak{p}}$ is a root for the $F_{S_{\mathfrak{p}}}$-module $\mathcal{M}_{\mathfrak{p}}$ for every prime ideal $\mathfrak{p} \subset S$, [Lyu97, Definition-Proposition 1.3]. Then,

$$
\begin{aligned}
\mu^{i}(\mathfrak{p}, \mathcal{M}) & =\mu^{i}(\mathfrak{p}, M) \text { by Lemma } 3.2 . \\
& =\operatorname{dim}_{S_{\mathfrak{p}} / \mathfrak{p} S_{\mathfrak{p}}} \operatorname{Ext}_{S_{\mathfrak{p}}}^{i}\left(S_{\mathfrak{p}} / \mathfrak{p} S_{\mathfrak{p}}, M_{\mathfrak{p}}\right) \\
& =\operatorname{dim}_{S_{\mathfrak{p}} / \mathfrak{p} S_{\mathfrak{p}}} \operatorname{Tor}_{\operatorname{dim} S_{\mathfrak{p}}-i}^{S_{\mathfrak{p}}}\left(S_{\mathfrak{p}} / \mathfrak{p} S_{\mathfrak{p}}, M_{\mathfrak{p}}\right) \text { by Remark 3.1. } \\
& \leq \operatorname{rank}\left(G_{\operatorname{dim} S_{\mathfrak{p}}-i}\right) \text { because } G_{\bullet} \otimes S_{\mathfrak{p}} \text { is a resolution for } M_{\mathfrak{p}} . \\
& =b_{\operatorname{dim} S_{\mathfrak{p}}-i} \\
& \leq B
\end{aligned}
$$

Hence, $\mu^{i}(\mathfrak{p}, \mathcal{M}) \leq B$, which proves the claim for $F$-finite $F$-modules. The claim about Lyubeznik numbers and local cohomology follows from the fact that $H_{I}^{n-i}(S)$ is always an $F$-finite $F$-module.

Remark 3.4. Under the notation of Theorem 3.3, we can take

$$
B=\max \left\{\operatorname{dim}_{S / \mathfrak{m}} \operatorname{Tor}_{i}^{S}\left(S / \mathfrak{m}, \operatorname{Ext}_{S}^{j}(S / I, S)\right) \mid \mathfrak{m} \text { is a maximal ideal of } S \text { and } i, j \in \mathbb{N}\right\}
$$

as a bound of the Lyubeznik numbers of $S / I$. Then, one can bound the Lyubeznik numbers of $S / I$ in terms of Betti numbers of its degeneracy modules, $\operatorname{Ext}_{S}^{j}(S / I, S)$. Puthenpurakal's bound for polynomial rings of characteristic zero is given by the multiplicity of $H_{I}^{i}(S)$ as holonomic $D_{K}(S)$-module [Put13]. The bound presented in Theorem 3.3 could be sharper. For example, if $f$ is a polynomial, we have that 3.0 .3 gives $B=1$ as a bound, which is sharp. The bound presented in [Put13] is bigger than or equal to $(\operatorname{deg}(f))^{n}$. Unlike the bound presented in [Put13], $B$ is shown to be a bound for the localization at every prime ideal. 


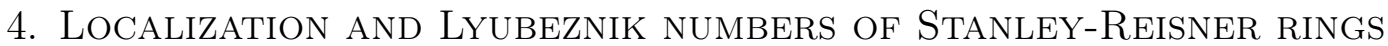

In this section, we study in deeper detail the Lyubeznik numbers of an Stanley-Reisner ring. In particular, we discuss a lower bound of $B$ presented in Theorem 3.3, under the assumption that $R$ is the localization of a Stanley-Reisner ring at its graded maximal ideal (see Theorem 4.4 for details).

Huneke and Sharp [HS93] and Lyubeznik [Lyu93] proved that the Bass numbers of local cohomology are finite. Motivated by this result and Theorem 3.3, one could expect that the Lyubeznik numbers, which are Bass numbers of local cohomology modules, would also resemble the behavior of Bass numbers of a finitely generated modules under localization (see 3.0.2). This is not true, even for the highest Lyubeznik number. The following example shows that the highest Lyubeznik number can both decrease and increase under localization.

Example 4.1. Let $S=K[x, y, z, u, v]$, and $I=(x, y) \cap(y, z) \cap(z, u) \cap(u, v)$. We have that $\operatorname{dim} R=4$, and the Hochster-Huneke graph of $R$ is connected. Then, we have that the highest Lyubeznik number, $\lambda_{4,4}(R)$, is 1 .

Let $\mathfrak{p}=(x, y, u, v)$. We have that the completion of $R_{\mathfrak{p}}$ is $L[[x, y, u, v]] / J$, where $J=$ $(x, y) \cap(u, v)$, where $L=R_{\mathfrak{p}} / \mathfrak{p} R_{\mathfrak{p}}$. Then $\operatorname{dim}\left(R_{\mathfrak{p}}\right)=3$ and the Hochster-Huneke of $R_{\mathfrak{p}}$ consists of two disconnected points.

Then, the highest Lyubeznik number of $R_{\mathfrak{p}}, \lambda_{3,3}\left(R_{\mathfrak{p}}\right)$, is 2 . If $\mathfrak{p}=(x, y)$, we have that its highest Lyubeznik number of $R_{\mathfrak{p}}$ is 1 .

In Remark 4.6, we present a general technique to build rings whose localization can have very different Lyubeznik numbers.

Remark 4.2. Àlvarez-Montaner and Vahidi have previously studied the behavior of Bass numbers of local cohomology over squarefree monomial ideals [ÀMV14, Section 5]. In particular, they studied the vanishing of these numbers. In addition, they presented examples that show different behavior of the Bass numbers of local cohomology modules and finitely generated modules.

Lemma 4.3. Let $S=K\left[x_{1}, \ldots, x_{n}\right]$ be a polynomial ring over a field. For a squarefree $S$-module $M$, we have

$$
\mathfrak{l}_{\sigma}\left(\operatorname{Ext}_{S}^{i}\left(M, \omega_{S}\right)\right) \cong \operatorname{Ext}_{K[\sigma]}^{i}\left(\mathfrak{l}_{\sigma}(M), \omega_{K[\sigma]}\right)
$$

Proof. In [Yan04, §3], the third author constructed the cochain complex

$$
\mathbf{D}_{S}(M): 0 \longrightarrow \mathbf{D}_{S}^{-n}(M) \longrightarrow \mathbf{D}_{S}^{-n+1}(M) \longrightarrow \cdots \longrightarrow \mathbf{D}_{S}^{0}(M) \longrightarrow 0
$$

with

$$
\mathbf{D}_{S}^{-i}(M)=\bigoplus_{\substack{\sigma \subset[n] \\ \# \sigma=i}}\left(M_{\sigma}\right)^{*} \otimes_{K} K[\sigma]
$$

such that $H^{-i}\left(\mathbf{D}_{S}(M)\right) \cong \operatorname{Ext}_{S}^{n-i}\left(M, \omega_{S}\right)$. Here $(-)^{*}$ means the $K$-dual of a vector space. 
We have

$$
\begin{aligned}
\mathfrak{l}_{\sigma}\left(\mathbf{D}_{S}^{-i}(M)\right) \cong \mathfrak{l}_{\sigma}\left(\bigoplus_{\substack{\tau \subset[n] \\
\# \tau=i}}\left(M_{\tau}\right)^{*} \otimes_{K} K[\tau]\right) \\
\cong \bigoplus_{\substack{\tau \subset[n] \\
\# \tau=i}}\left(M_{\tau}\right)^{*} \otimes_{K} \mathfrak{l}_{\sigma}(K[\tau]) \\
\cong \bigoplus_{\substack{\sigma^{c} \subset \tau \subset[n] \\
\# \tau=i}}\left(M_{\tau}\right)^{*} \otimes_{K} K[\tau \cap \sigma] \\
\cong \bigoplus_{\substack{\tau \subset \sigma \\
\# \tau=i-\sigma^{\mathrm{c}}}}\left(\mathfrak{l}_{\sigma}(M)_{\tau}\right)^{*} \otimes_{K} K[\tau] \\
\cong \mathbf{D}_{K[\sigma]}^{-i+\# \sigma^{\mathrm{c}}}\left(\mathfrak{l}_{\sigma}(M)\right) .
\end{aligned}
$$

These isomorphisms commute with the differential maps, and we have an isomorphism

$$
\mathfrak{l}_{\sigma}\left(\mathbf{D}_{S}^{\bullet}(M) \cong \mathbf{D}_{K[\sigma]}^{\bullet+\# \sigma^{\mathrm{c}}}\left(\mathfrak{l}_{\sigma}(M)\right)\right.
$$

of cochain complexes. Hence the assertion follows from the following computation

$$
\begin{aligned}
\mathfrak{l}_{\sigma}\left(\operatorname{Ext}_{S}^{n-i}\left(M, \omega_{S}\right)\right) & \cong \mathfrak{l}_{\sigma}\left(H^{-i}\left(\mathbf{D}_{S}^{\bullet}(M)\right)\right) \\
& \cong H^{-i}\left(\mathfrak{l}_{\sigma}\left(\mathbf{D}_{S}^{\bullet}(M)\right)\right) \\
& \cong H^{-i+\# \sigma^{\mathrm{c}}}\left(\mathbf{D}_{K[\sigma]}^{\bullet}\left(\mathfrak{l}_{\sigma}(M)\right)\right) \\
& \cong \operatorname{Ext}_{K[\sigma]}^{\# \sigma-i+\# \sigma^{\mathrm{c}}}\left(\mathfrak{l}_{\sigma}(M), \omega_{K[\sigma]}\right) \\
& \cong \operatorname{Ext}_{K[\sigma]}^{n-i}\left(\mathfrak{l}_{\sigma}(M), \omega_{\sigma}\right)
\end{aligned}
$$

The following is a generalization of Equation 2.2.1.

Theorem 4.4. Let $S=K\left[x_{1}, \ldots, x_{n}\right]$ be a polynomial ring over a field. Let $\Delta \subset 2^{[n]}$ be a simplicial complex.

$$
\lambda_{i, j}\left(K[\Delta]_{\mathfrak{p}_{\sigma}}\right)=\operatorname{dim}_{K}\left[\operatorname{Ext}_{S}^{\# \sigma-i}\left(\operatorname{Ext}_{S}^{\# \sigma-j}\left(K[\Delta], \omega_{S}\right), \omega_{S}\right)\right]_{\sigma^{c}} .
$$

Proof. By Lemma 4.3, we ave

$$
\begin{aligned}
\mathfrak{l}_{\sigma}\left(\operatorname{Ext}_{S}^{i}\left(\operatorname{Ext}_{S}^{j}\left(K[\Delta], \omega_{S}\right), \omega_{S}\right)\right) & \cong \operatorname{Ext}_{K[\sigma]}^{i}\left(\mathfrak{l}_{\sigma}\left(\operatorname{Ext}_{S}^{j}\left(K[\Delta], \omega_{S}\right)\right), \omega_{K[\sigma]}\right) \\
& \cong \operatorname{Ext}_{K[\sigma]}^{i}\left(\operatorname{Ext}_{K[\sigma]}^{j}\left(\mathfrak{l}_{\sigma}(K[\Delta]), \omega_{K[\sigma]}\right), \omega_{K[\sigma]}\right) \\
& \cong \operatorname{Ext}_{K[\sigma]}^{i}\left(\operatorname{Ext}_{K[\sigma]}^{j}\left(K\left[\mathrm{lk}_{\Delta} \sigma^{\mathrm{c}}\right], \omega_{K[\sigma]}\right), \omega_{K[\sigma]}\right)
\end{aligned}
$$

Hence we have

$$
\left[\operatorname{Ext}_{S}^{i}\left(\operatorname{Ext}_{S}^{j}\left(K[\Delta], \omega_{S}\right), \omega_{S}\right)\right]_{\sigma^{\mathrm{c}}} \cong\left[\operatorname{Ext}_{K[\sigma]}^{i}\left(\operatorname{Ext}_{K[\sigma]}^{j}\left(K\left[\mathrm{lk}_{\Delta} \sigma^{\mathrm{c}}\right], \omega_{K[\sigma]}\right), \omega_{K[\sigma]}\right)\right]_{0} .
$$

Combining this fact with (2.2.2), we are done.

The next result easily follows from the above theorem. 
Corollary 4.5. Let $S=K\left[x_{1}, \ldots, x_{n}\right]$ be a polynomial ring over a field, and $\mathfrak{m}=\left(x_{1}, \ldots, x_{n}\right)$. Let $\Delta \subset 2^{[n]}$ be a simplicial complex. If $R$ is the localization $K[\Delta]_{\mathfrak{m}}$ of a Stanley-Reisner ring $K[\Delta]=S / I_{\Delta}$, the number $B$ presented in Theorem 3.3 must satisfy

$$
B \geq \operatorname{dim}_{K}\left[\operatorname{Ext}_{S}^{i}\left(\operatorname{Ext}_{S}^{j}\left(K[\Delta], \omega_{S}\right), \omega_{S}\right)\right]_{\sigma}
$$

for all $\sigma \subset[n]$ and all $i, j$.

Remark 4.6. (1) It is easy to connect Corollary 4.5 with the argument in $\S 3$. In fact, if $\cdots \rightarrow S^{b_{i}} \rightarrow \cdots \rightarrow S^{b_{0}} \rightarrow 0$ is a free resolution of $\operatorname{Ext}_{S}^{j}\left(K[\Delta], \omega_{S}\right)$, then we have

$$
b_{i} \geq \operatorname{dim}_{K}\left[\operatorname{Ext}_{S}^{i}\left(\operatorname{Ext}_{S}^{j}\left(K[\Delta], \omega_{S}\right), \omega_{S}\right)\right]_{\sigma} .
$$

(2) Let $I_{\Delta} \subset S=K\left[x_{1}, \ldots, x_{n}\right]$ be a Stanley-Reisner ideal, and set $S^{\prime}:=S\left[x_{n+1}\right]=$ $K\left[x_{1}, \ldots, x_{n+1}\right]$. Consider the monomial ideal $I^{\prime}:=I_{\Delta} S^{\prime} \cap\left(x_{n+1}\right)$ of $S^{\prime}$. Since the codimension of $I^{\prime}$ is $1, S^{\prime} / I^{\prime}$ (more precisely, its localization at the graded maximal ideal of) has the trivial Lyubeznik table as shown in [ĀMY14]. However, for a prime ideal $\mathfrak{p}:=\left(x_{1}, \ldots, x_{n}\right)$ of $S^{\prime}$, we have

$$
\lambda_{i, j}\left(\left(S^{\prime} / I^{\prime}\right)_{\mathfrak{p}}\right)=\lambda_{i, j}\left(S / I_{\Delta}\right) .
$$

Hence the Lyubeznik table of $\left(S^{\prime} / I^{\prime}\right)_{\mathfrak{p}}$ can be far from trivial.

Besides the bad behavior under localization of the Lyubeznik numbers, the generalized Lyubeznik numbers and the multiplicities of local cohomology behave as expected with respect to localization.

Proposition 4.7. Let $S=K\left[x_{1}, \ldots, x_{n}\right]$ be a polynomial ring over a field. Let $I \subset S$ denote an squarefree monomial ideal and $d=\operatorname{dim}(S / I)$ and $\mathfrak{m}=\left(x_{1}, \ldots, x_{n}\right)$. Let $\mathfrak{p} \subset S$ denote any prime ideal containing $I$, and $h=\operatorname{dim}(S / I)-\operatorname{dim}\left(S_{\mathfrak{p}} / I S_{\mathfrak{p}}\right)$. Then,

$$
\lambda_{0}^{j-h}\left(S_{\mathfrak{p}} / I S_{\mathfrak{p}}\right) \leq \lambda_{0}^{j}(S / I)
$$

for $j \in \mathbb{N}$. Moreover,

$$
\gamma_{i-h, j-h}\left(S_{\mathfrak{p}} / I S_{\mathfrak{p}}\right) \leq \gamma_{i, j}(S / I)
$$

Proof. We fix $j \in \mathbb{N}$. Since $I$ is a monomial ideal, there exists a filtration of $D_{K}(S)$-modules

$$
0=M_{0} \subset M_{1} \subset M_{2} \subset \ldots \subset M_{\ell}=H_{I}^{n-j}(S)
$$

such that $M_{t} / M_{t-1} \cong H_{\mathfrak{p}_{\alpha}}^{|\alpha|}(S)$ for some $\alpha \subset[n]$ [Yan01, Proposition 2.10 and Remark 2.12]. We recall that

$$
m_{j,-\alpha}=\#\left\{t \mid M_{t} / M_{t-1} \cong H_{\mathfrak{p}_{\alpha}}^{|\alpha|}(S)\right\} .
$$

We note that $H_{\mathfrak{p}_{\alpha}}^{|\alpha|}(R)$ is a simple $D_{K}(R)$-module, and so, length $D_{K}(S) H_{I}^{n-j}(S)=\sum_{\alpha} m_{n-j,-\alpha}$.

Let $A=\widehat{S_{\mathfrak{p}}}$ and $L=S_{\mathfrak{p}} / \mathfrak{p} S_{\mathfrak{p}}$. By Cohen Structure Theorems, $A$ is a power series ring over $L$. We have that for every variable $x_{r} \in S, x_{r}$ is either a unit or a regular element in $A$. Hence, $I A$ is still induced from a squarefree monomial ideal. We note that $A$ is a flat extension of $S$ and that $H_{I}^{n-j}(S) \otimes_{S} A \cong H_{I}^{n-j}(A)$ as $D_{L}(A)$-modules. In addition,

$$
0=M_{0} \otimes_{S} A \subset M_{1} \otimes_{S} A \subset M_{2} \otimes_{S} A \subset \ldots \subset M_{\ell} \otimes_{A}=H_{I}^{n-j}(A)
$$

gives a filtration of $D_{L}(A)$-modules such that $M_{t} \otimes_{S} A / M_{t-1} \otimes_{S} A \cong H_{\mathfrak{p}_{\alpha} A}^{|\alpha|}(A)$ is either a simple $D_{L}(A)$-module or zero (depending on whether $\mathfrak{p}_{\alpha} \subset \mathfrak{p}$ ). Let

$$
m_{-\alpha}^{\prime}=\#\left\{t \mid M_{t} / M_{t-1} \otimes_{S} A \cong H_{\mathfrak{p}_{\alpha}}^{|\alpha|}(A) \text { and } \mathfrak{p}_{\alpha} \subset \mathfrak{p}\right\} \text {. }
$$


We note that $m_{-\alpha}^{\prime} \leq m_{n-j,-\alpha}$. Hence,

$$
\begin{aligned}
\lambda_{0}^{j-h}\left(S_{\mathfrak{p}} / I S_{\mathfrak{p}}\right) & =\operatorname{length}_{D_{L}\left(S_{\mathfrak{p}}\right)} H_{I}^{\operatorname{dim}\left(S_{\mathfrak{p}}\right)-j+h}\left(S_{\mathfrak{p}}\right) \\
& =\operatorname{length}_{D_{L}\left(S_{\mathfrak{p}}\right)} H_{I}^{n-j}\left(S_{\mathfrak{p}}\right) \\
& =\sum_{\alpha} m_{-\alpha}^{\prime} \\
& \leq \sum_{\alpha} m_{n-j,-\alpha} \\
& =\operatorname{length}_{D_{K}(S)} H_{I}^{\operatorname{dim}(S)-h-j}(S) \\
& =\lambda_{0}^{j+h}\left(S_{\mathfrak{m}} / I S_{\mathfrak{m}}\right) .
\end{aligned}
$$

Moreover,

$$
\gamma_{i-h, j-h}\left(S_{\mathfrak{p}} / I S_{\mathfrak{p}}\right)=\sum_{|\alpha|=n-i} n_{\alpha} \leq \sum_{|\alpha|=n-i} m_{n-i, \alpha}=\gamma_{i, j}(S / I)
$$

Remark 4.8. As noted in Remark 2.20, the length $H_{I_{\Delta}}^{i}(R)$ as a $D_{K}(S)$ is determined by the $\mathbb{Z}^{n}$-graded Betti numbers of the Alexander dual of $I_{\Delta}$. The same applies to the invariants $\gamma_{i, j}$. We point out that from this characterization, it does not follow that the generalized Lyubeznik number cannot increase under localization. This is because the multigrading is lost after localization; for instance, the linear strands of a graded free resolution are not preserved.

\section{Polarization and Lyubeznik numbers of Stanley-Reisner Rings}

The main theorem in this section we prove that the (original) Lyubeznik numbers given by a monomial ideal and its polarization are essentially the same.

Theorem 5.1. Let $S=K\left[x_{1}, \ldots, x_{n}\right]$ be a polynomial ring, $\mathfrak{m}=\left(x_{1}, \ldots, x_{n}\right)$ and $I \subset S$ be a monomial ring. Let $\widetilde{I}$ denote the polarization of $I$, and $\widetilde{S}=K\left[x_{r, s}\right]$ denote the polarization ring. Let $\mathfrak{n}$ be the maximal homogeneous ideal of $\widetilde{S}$ and $h=\operatorname{dim}(\widetilde{S} / \widetilde{I})-\operatorname{dim}(S / I)$. Then,

$$
\lambda_{i-h, j-h}\left(S_{\mathfrak{m}} / I S_{\mathfrak{m}}\right)=\lambda_{i, j}\left(\widetilde{S}_{\mathfrak{n}} / \widetilde{I}_{\mathfrak{n}}\right) .
$$

for every $i, j \in \mathbb{N}$.

Proof. It suffices to prove that

$$
\left(\operatorname{Ext}_{S}^{i}\left(\operatorname{Ext}_{S}^{j}\left(S / \sqrt{I}, \omega_{S}\right), \omega_{S}\right)\right)_{\mathbf{0}} \cong\left(\operatorname{Ext}_{\widetilde{S}}^{i}\left(\operatorname{Ext}_{\widetilde{S}}^{j}\left(\widetilde{S} / \widetilde{I}, \omega_{\widetilde{S}}\right), \omega_{\widetilde{S}}\right)\right)_{\mathbf{0}}
$$

for all $i, j$ (the role of $i, j$ is different from that in the original statement). We start from the following computation.

$$
\begin{aligned}
\operatorname{Ext}_{\widetilde{S}}^{i}\left(\operatorname{Ext}_{\widetilde{S}}^{j}\left(\widetilde{S} / \widetilde{I}, \omega_{\widetilde{S}}\right), \omega_{\widetilde{S}}\right) & \cong \operatorname{Ext}_{\widetilde{S}}^{i}\left(\operatorname{Ext}_{\widetilde{S}}^{j}\left(\operatorname{pol}_{\alpha}(S / I), \omega_{\widetilde{S}}\right), \omega_{\widetilde{S}}\right) \\
& \cong \operatorname{Ext}_{\widetilde{S}}^{i}\left(\operatorname{pol}^{\alpha}\left(\operatorname{Ext}_{S}^{j}(S / I, S(-\alpha))\right), \omega_{\widetilde{S}}\right) \\
& \cong \operatorname{pol}_{\alpha}\left(\operatorname{Ext}_{S}^{i}\left(\operatorname{Ext}_{S}^{j}(S / I, S(-\alpha)), S(-\alpha)\right)\right) \\
& \cong \operatorname{pol}_{\alpha}\left(\operatorname{Ext}_{S}^{i}\left(\operatorname{Ext}_{S}^{j}\left(S / I, \omega_{S}\right), \omega_{S}\right)\right),
\end{aligned}
$$


where the second and the third isomorphisms follow from [Yan12, Theorem 4.4]. Hence we have

$$
\left(\operatorname{Ext}_{\widetilde{S}}^{i}\left(\operatorname{Ext}_{\widetilde{S}}^{j}\left(\widetilde{S} / \widetilde{I}, \omega_{\widetilde{S}}\right), \omega_{\widetilde{S}}\right)\right)_{\mathbf{0}} \cong\left(\operatorname{Ext}_{S}^{i}\left(\operatorname{Ext}_{S}^{j}\left(S / I, \omega_{S}\right), \omega_{S}\right)\right)_{\mathbf{0}}
$$

For a $\mathbb{Z}^{n}$-graded module $M=\bigoplus_{\beta \in \mathbb{Z}^{n}} M_{\beta}$, set $M_{\succeq \mathbf{0}}=\bigoplus_{\beta \succeq \mathbf{0}} M_{\beta}$. Then, [EO13, Theorem 2.3 (1) (a)] states that

$$
\left(\operatorname{Ext}_{S}^{j}\left(S / I, \omega_{S}\right)\right)_{\succeq \mathbf{0}} \cong \operatorname{Ext}_{S}^{j}\left(S / \sqrt{I}, \omega_{S}\right) .
$$

Ene and Okazaki [EO13] constructed two exact functors $\mathfrak{r}^{*}:{ }^{*} \bmod _{\alpha} S \rightarrow \operatorname{Sq} S$ and $\mathfrak{s}^{*}$ : ${ }^{*} \bmod _{\alpha} S \rightarrow \operatorname{Sq} S$. Here $\mathfrak{r}^{*}$ is the "radical functor" sending a monomial ideal $I$ (resp. $S / I$ ) to $\sqrt{I}(\operatorname{resp} . S / \sqrt{I})$, and $\mathfrak{s}^{*}$ is just defined by $\mathfrak{s}^{*}(M)=[M(\alpha-\mathbf{1})]_{\succeq \mathbf{0}}$. Now we have

$$
\begin{aligned}
\mathfrak{r}^{*}\left(\operatorname{Ext}_{S}^{i}\left(\operatorname{Ext}_{S}^{j}\left(S / I, \omega_{S}\right), \omega_{S}\right)\right) & \cong \mathfrak{r}^{*}\left(\operatorname{Ext}_{S}^{i}\left(\operatorname{Ext}_{S}^{j}(S / I, S(-\alpha)), S(-\alpha)\right)\right) \\
& \cong \operatorname{Ext}_{S}^{i}\left(\mathfrak{s}^{*}\left(\operatorname{Ext}_{S}^{j}(S / I, S(-\alpha))\right), \omega_{S}\right) \\
& \cong \operatorname{Ext}_{S}^{i}\left(\operatorname{Ext}_{S}^{j}\left(S / \sqrt{I}, \omega_{S}\right), \omega_{S}\right),
\end{aligned}
$$

where the second isomorphism follows from [EO13, Theorem 2.3 (1) (b)], and the third one follows from (5.0.5) (recall that $\omega_{S}=S(-\mathbf{1})$ ). By the construction of $\mathfrak{r}^{*}$, we have

$$
\left(\operatorname{Ext}_{S}^{i}\left(\operatorname{Ext}_{S}^{j}\left(S / I, \omega_{S}\right), \omega_{S}\right)\right)_{\mathbf{0}} \cong\left(\operatorname{Ext}_{S}^{i}\left(\operatorname{Ext}_{S}^{j}\left(S / \sqrt{I}, \omega_{S}\right), \omega_{S}\right)\right)_{\mathbf{0}} .
$$

Combining this with (5.0.4), we have the expected isomorphism.

Remark 5.2. Theorem 5.1 is a delicate result in the following sense. For example, set

$$
I:=\left(x^{2} y, x^{2} z, x y z, x z^{2}, y^{3}, y^{2} z, y z^{2}\right) \subset S:=\mathbb{k}[x, y, z]
$$

and

$$
I^{\prime}:=\left(x_{1} x_{2} y_{3}, x_{1} x_{2} z_{3}, x_{1} y_{2} z_{3}, x_{1} z_{2} z_{3}, y_{1} y_{2} y_{3}, y_{1} y_{2} z_{3}, y_{1} z_{2} z_{3}\right) \subset \widetilde{S}:=\mathbb{k}\left[x_{i}, y_{i}, z_{i} \mid i=1,2,3\right] .
$$

Then $\Theta$ forms an $\widetilde{S} / I^{\prime}$-regular sequence, and gives an isomorphism $\widetilde{S} /\left(I^{\prime}+(\Theta)\right) \cong S / I$ (see last part of Subsection 2.2 to recall notation). In this sense, $I^{\prime}$ is a "non-standard" polarization of $I$. We see that $S / I$ is sequentially Cohen-Macaulay, and has a trivial Lyubeznik table [ÀM14, ÀMY14]. However, we have

$$
\lambda_{i, j}\left(\widetilde{S} / I^{\prime}\right)= \begin{cases}2 & \text { if } i=j=7 \\ 1 & \text { if } i=5, j=6 \\ 0 & \text { otherwise. }\end{cases}
$$

Hence a non-standard polarization may not preserve the Lyubeznik table.

Remark 5.3. Under the Notation of Theorem 5.1, we can give a different proof of

$$
\lambda_{d, d}(S / I)=\lambda_{d^{\prime}, d^{\prime}}(\widetilde{S} / \widetilde{I}) .
$$

by comparing the Hochster-Huneke graph of $S / I$ and $\widetilde{S} / \widetilde{I}$. The highest Lyubeznik number for the localization of an Stanley-Reisner ring at the maximal homogeneous ideal is equal to the number of the connected components of the Hochster-Huneke graph by Remark 2.6. The vertices of the Hochster-Huneke graph are the minimal primes of minimum height. Let $\left\{g_{1}, \ldots, g_{l}\right\}$ be the set of minimal monomial generators of $I$ and let $\widetilde{g}_{i}$ be the polarization of $m_{i}$. Then $\left\{\widetilde{g}_{1}, \ldots, \widetilde{g}_{l}\right\}$ is the set of minimal monomial generators of $\widetilde{I}$. 
We make two observations. First, $\left(x_{i_{1}}, \ldots, x_{i_{r}}\right)$ is a vertex in the Hochster graph of $I$ if and only if $\left(x_{i_{1}, 1}, \ldots, x_{i_{r}, 1}\right)$ is a vertex of the Hochster-Huneke graph of $\widetilde{I}$. Second, if $\left(x_{i_{1}, c_{1}}, \ldots, x_{i_{r}, c_{r}}\right)$ is a vertex of the Hochster-Huneke graph of $\widetilde{I}$, then $\left(x_{i_{1}, b_{1}}, \ldots, x_{i_{r}, b_{r}}\right)$ is a vertex of Hochster-Huneke graph of $\widetilde{I}$ for all $b_{j} \leq c_{j}$.

We note that $\left(x_{i_{1}}, \ldots, x_{i_{r}}\right)$ and $\left(x_{j_{1}}, \ldots, x_{j_{r}}\right)$ are connected to each other by an edge if and only if $\left(x_{i_{1}, 1}, \ldots, x_{i_{r}, 1}\right)$ and $\left(x_{j_{1}, 1}, \ldots, x_{j_{r}, 1}\right)$ are connected to each other by an edge. In addition, $\left(x_{i_{1}, 1}, \ldots, x_{i_{r}, 1}\right)$ and $\left(x_{i_{1}, c_{1}}, \ldots, x_{i_{r}, c_{r}}\right)$ are connected via the following paths in Hochster-Huneke graph:

$$
\begin{gathered}
\left(x_{i_{1}, 1}, x_{i_{2}, 1}, \ldots, x_{i_{r}, 1}\right),\left(x_{i_{1}, 2}, x_{i_{2}, 1}, \ldots, x_{i_{r}, 1}\right), \ldots,\left(x_{i_{1}, c_{1}}, x_{i_{2}, 1}, \ldots, x_{i_{r}, 1}\right) \\
\left(x_{i_{1}, c_{1}}, x_{i_{2}, 1}, \ldots, x_{i_{r}, 1}\right),\left(x_{i_{1}, c_{1}}, x_{i_{2}, 2}, \ldots, x_{i_{r}, 1}\right), \ldots,\left(x_{i_{1}, c_{1}}, x_{i_{2}, c_{2}}, \ldots, x_{i_{r}, 1}\right) \\
\vdots \\
\left(x_{i_{1}, c_{1}}, \ldots, x_{i_{r-1}, c_{r-1}}, x_{i_{r}, 1}\right),\left(x_{i_{1}, c_{1}}, \ldots, x_{i_{r-1}, c_{r-1}}, x_{i_{r}, 2}\right), \ldots,\left(x_{i_{1}, c_{1}}, \ldots, x_{i_{r-1}, c_{r-1}}, x_{i_{r}, c_{r}}\right) .
\end{gathered}
$$

By the previous assertions, we get that there is an bijective correspondence between connected components of the two Hochster-Huneke graphs under discussion, which sends the connected component of $\left(x_{i_{1}, c_{1}}, \ldots, x_{i_{r}, c_{r}}\right)$ to the connected component of $\left(x_{i_{1}}, \ldots, x_{i_{r}}\right)$.

Proposition 5.4. Let $S=K\left[x_{1}, \ldots, x_{n}\right]$ be a polynomial ring and $I \subset S$ be a monomial ring. Let $\widetilde{I}$ denote the polarization of $I$, and $\widetilde{S}=K\left[x_{r, s}\right]$ denote the polarization ring. Let $h=\operatorname{dim}(\widetilde{S} / \widetilde{I})-\operatorname{dim}(S / I)$. Then,

$$
\lambda_{0}^{j-h}(S / \sqrt{I}) \leq \lambda_{0}^{j}(\widetilde{S} / \widetilde{I}) .
$$

Moreover,

$$
\gamma_{i-h, j-h}(S / I) \leq \gamma_{i, j}(\widetilde{S} / \widetilde{I})
$$

Proof. We fix $j \in \mathbb{N}$. Let $\mathfrak{m}=\left(x_{1}, \ldots, x_{n}\right)$ denote the maximal homogeneous ideal of $R$. Since $I$ is a monomial ideal, there exists a filtration of $D_{K}(S)$-modules

$$
0=M_{0} \subset M_{1} \subset M_{2} \subset \ldots \subset M_{\ell}=H_{I}^{n-j}(S)
$$

such that $M_{t} / M_{t-1} \cong H_{\mathfrak{p}_{t}}^{\mathrm{ht}\left(\mathfrak{p}_{t}\right)}(S)$ where $\mathfrak{p}_{t}$ is a prime ideal generated by a subset of $\left\{x_{1}, \ldots, x_{n}\right\}$ [Yan01, Proposition 2.10 and Remark 2.12]. We recall that

$$
m_{n-j,-\alpha}=\#\left\{t \mid M_{t} / M_{t-1} \cong H_{\mathfrak{p}_{\alpha}}^{|\alpha|}(S)\right\} .
$$

We note that $H_{\mathfrak{p}_{t}}^{\mathrm{ht}\left(\mathfrak{p}_{t}\right)}(R)$ is a simple $D_{K}(S)$ for every $t=1, \ldots, \ell$, and so, length ${ }_{D_{K}(S)} H_{I}^{n-j}(S)=$ $\sum_{\alpha} m_{n-j,-\alpha}=\ell$. Let $Q=m \widetilde{S}, A=\widehat{\widetilde{S}_{Q}}$ and $L=A / Q A$. We note that the induced map $S \rightarrow \widetilde{S} \rightarrow A$ makes $A$ a flat extension of $S$. We have that

$$
H_{I}^{n-j}(S) \otimes_{S} A \cong H_{I}^{n-j}(A)
$$

is a $D_{L}(A)$-module. In addition,

$$
0=M_{0} \otimes_{S} A \subset M_{1} \otimes_{S} A \subset M_{2} \otimes_{S} A \subset \ldots \subset M_{\ell}=H_{I}^{n-j}(A),
$$

gives a filtration of $D_{F}(A)$-modules such that $M_{t} \otimes_{S} A / M_{t-1} \otimes_{S} A \cong H_{\mathfrak{p}_{t}}^{\text {ht } \mathfrak{p}_{t}}(A) \neq 0$ is a simple $D_{L}(A)$-module. Hence,

$$
m_{n-i,-\alpha}=\#\left\{t \mid M_{t} / M_{t-1} \otimes_{S} A \cong H_{\mathfrak{p}_{\alpha}}^{|\alpha|}(A)\right\} .
$$


As before, there exists a filtration of $D_{K}(\widetilde{S})$-modules

$$
0=M_{0}^{\prime} \subset M_{1}^{\prime} \subset M_{2}^{\prime} \subset \ldots \subset M_{\ell^{\prime}}^{\prime}=H_{\widetilde{I}}^{n-j}(\widetilde{S})
$$

such that $M_{t}^{\prime} / M_{t-1}^{\prime} \cong H_{\mathfrak{p}_{t}^{\prime}}^{\mathrm{ht} \mathfrak{p}_{t}^{\prime}}(\widetilde{S})$ where $\mathfrak{p}_{t}^{\prime}$ is a prime ideal generated by a subset of $\left\{x_{r, s}\right\}$ [Yan01, Proposition 2.10 and Remark 2.12]. We note that $H_{\mathfrak{p}_{t}^{\prime}}^{\text {ht } \mathfrak{p}_{t}^{\prime}}(\widetilde{S})$ is a simple $D_{L}(\widetilde{S})$ module, and so, $\operatorname{length}_{D_{K}(\widetilde{S})} H_{\widetilde{I}}^{i}(\widetilde{S})=\ell^{\prime}$. We have that $A$ is a flat $\widetilde{S}$-algebra and

$$
H_{\widetilde{I}}^{i}(\widetilde{S}) \otimes_{\widetilde{S}} A \cong H_{I}^{n-j}(A)
$$

is a $D_{L}(A)$-module. As before, we have that

$$
0=M_{0}^{\prime} \otimes_{\widetilde{S}} A \subset M_{1}^{\prime} \otimes_{\widetilde{S}} A \subset M_{2}^{\prime} \otimes_{\widetilde{S}} A \subset \ldots \subset M_{\ell^{\prime}}^{\prime} \otimes_{\widetilde{S}} A=H_{\widetilde{I}}^{i}(A),
$$

gives a filtration of $D_{L}(A)$-modules. We have that $M_{t \otimes_{S} A}^{\prime} / M_{t-1}^{\prime} \otimes_{\widetilde{S}} A \neq 0$ if and only if $\mathfrak{p}_{t}^{\prime} \subset\left\{x_{1,1}, \ldots, x_{n, 1}\right\}$, and if that is the case, $M_{t}^{\prime} / M_{t-1}^{\prime} \otimes_{\widetilde{S}} A \cong H_{\mathfrak{p}_{t}^{\prime}}^{\left|\mathfrak{p}^{\prime}\right|}(A)$ is a simple $D_{L}(A)$ module. Hence, if $m_{-\alpha}^{\prime}=\#\left\{t \mid M_{t} / M_{t-1} \otimes_{S} A \cong H_{\mathfrak{p}_{\alpha}}^{|\alpha|}(A)\right.$ and $\left.\mathfrak{p}_{\alpha} \subset Q\right\}$, we have that $m_{-\alpha}^{\prime} \leq m_{n-j,-\alpha}$. Hence,

$$
\begin{aligned}
\lambda_{0}^{j+h}(\widetilde{S} / \widetilde{I}) & =\operatorname{length}_{D_{K}(\widetilde{S})} H_{I}^{\operatorname{dim}(\widetilde{S})-j-h}(\widetilde{S}) \\
& =\operatorname{length}_{D_{L}(\widetilde{S})} H_{\widetilde{I}}^{n-j-h}(\widetilde{S}) \\
& =\sum_{\alpha} m_{-\alpha}^{\prime} \\
& \leq \sum_{\alpha} m_{n-j,-\alpha} \\
& =\operatorname{length}_{D_{K}(S)} H_{I}^{n-j}(S) \\
& =\lambda_{0}^{j}\left(S_{\mathfrak{m}} / I S_{\mathfrak{m}}\right) .
\end{aligned}
$$

Moreover,

$$
\gamma_{i+h, j+h}(\widetilde{S} / \widetilde{I})=\sum_{|\alpha|=n-j} m_{-\alpha}^{\prime} \leq \sum_{|\alpha|=n-j} m_{n-j,-\alpha}=\gamma_{i, j}(S / I)
$$

Remark 5.5. As noted in Remark 2.20, the generalized Lyubeznik numbers and invariants $\gamma_{i, j}$ are given by adding multigraded Betti numbers of $I^{\vee}$. A corollary of the proof for Proposition 5.4 is that

$$
\beta_{i,-\alpha}\left((\sqrt{I})^{\vee}\right) \leq \beta_{i,-(\alpha, 0, \ldots, 0)}\left((\widetilde{I})^{\vee}\right)
$$

where $(\alpha, 0, \ldots, 0)$ is the extension of the exponent $\alpha$ given the inclusion $S \rightarrow \widetilde{S}$ given by $x_{j} \mapsto x_{j, 1}$. We point out that this corollary is not a consequence of the fact that

$$
\beta_{i,-\alpha}\left((\sqrt{I}) \leq \beta_{i,-\alpha}\left((I)=\beta_{i,-(\alpha, 0, \ldots, 0)}((\widetilde{I}),\right.\right.
$$

which follows from [EO13, Theorem 1.4] and [Pee11, Theorem 2.14]. 


\section{Acknowledgments}

We thank Josep Àlvarez-Montaner, Craig Huneke, and Ryota Okazaki for helpful comments and suggestions. The first author was partially supported by NSF Grant DMS1259142. The second author was partially supported by the Council for Science and Technology of Mexico, CONACYT 207063. The third author was partially supported by JSPS KAKENHI 25400057.

\section{REFERENCES}

[ÅM04] Josep Àlvarez Montaner. Some numerical invariants of local rings. Proc. Amer. Math. Soc., 132(4):981-986 (electronic), 2004. 1, 3, 7

[ÅM14] Josep Àlvarez Montaner. Lyubeznik table of sequentially Cohen-Macaulay rings. To Appear in Communications in Algebra, 2014. 13

[ÀMGLZA03] Josep Àlvarez Montaner, Ricardo García López, and Santiago Zarzuela Armengou. Local cohomology, arrangements of subspaces and monomial ideals. Adv. Math., 174(1):35-56, 2003. 3,7

[ÀMV14] J. Àlvarez Montaner and A. Vahidi. Lyubeznik numbers of monomial ideals. Trans. Amer. Math. Soc., 366(4):1829-1855, 2014. 3, 9

[ÀMY14] J. Àlvarez Montaner and K. Yanagawa. "lyubeznik numbers of local rings and linear strands of graded ideal. Preprint, arXiv:1409.6486 [math.AC], 2014. 11, 13

[BB05] Manuel Blickle and Raphael Bondu. Local cohomology multiplicities in terms of étale cohomology. Ann. Inst. Fourier (Grenoble), 55(7):2239-2256, 2005. 1

[Bjö72] Jan-Erik Björk. The global homological dimension of some algebras of differential operators. Invent. Math., 17:67-78, 1972. 6

[Bjö79] Jan-Erik Björk. Rings of differential operators, volume 21 of North-Holland Mathematical Library. North-Holland Publishing Co., Amsterdam, 1979. 6

[BS98] Markus P. Brodmann and Rodney Y. Sharp. Local cohomology: an algebraic introduction with geometric applications, volume 60 of Cambridge Studies in Advanced Mathematics. Cambridge University Press, Cambridge, 1998. 2

[Cou95] S. C. Coutinho. A primer of algebraic D-modules, volume 33 of London Mathematical Society Student Texts. Cambridge University Press, Cambridge, 1995. 6

[EO13] Viviana Ene and Ryota Okazaki. On the radical of multigraded modules. J. Algebra, 388:1021, 2013. 13, 15

[Far06] Sara Faridi. Monomial ideals via square-free monomial ideals. In Commutative algebra, volume 244 of Lect. Notes Pure Appl. Math., pages 85-114. Chapman \& Hall/CRC, Boca Raton, FL, 2006. 3

[GLS98] R. García López and C. Sabbah. Topological computation of local cohomology multiplicities. Collect. Math., 49(2-3):317-324, 1998. Dedicated to the memory of Fernando Serrano. 1

[HH94] Melvin Hochster and Craig Huneke. Indecomposable canonical modules and connectedness. In Commutative algebra: syzygies, multiplicities, and birational algebra (South Hadley, MA, 1992), volume 159 of Contemp. Math., pages 197-208. Amer. Math. Soc., Providence, RI, 1994. 3

[HH11] Jürgen Herzog and Takayuki Hibi. Monomial ideals, volume 260 of Graduate Texts in Mathematics. Springer-Verlag London, Ltd., London, 2011. 3

[HS93] Craig L. Huneke and Rodney Y. Sharp. Bass numbers of local cohomology modules. Trans. Amer. Math. Soc., 339(2):765-779, 1993. 2, 9

$\left[\mathrm{ILL}^{+} 07\right] \quad$ Srikanth B. Iyengar, Graham J. Leuschke, Anton Leykin, Claudia Miller, Ezra Miller, Anurag K. Singh, and Uli Walther. Twenty-four hours of local cohomology, volume 87 of Graduate Studies in Mathematics. American Mathematical Society, Providence, RI, 2007. 2

[Lyu93] Gennady Lyubeznik. Finiteness properties of local cohomology modules (an application of $D$-modules to commutative algebra). Invent. Math., 113(1):41-55, 1993. 1, 2, 9

[Lyu97] Gennady Lyubeznik. $F$-modules: applications to local cohomology and $D$-modules in characteristic $p>0$. J. Reine Angew. Math., 491:65-130, 1997. 5, 6, 8 
[Lyu00] Gennady Lyubeznik. Finiteness properties of local cohomology modules: a characteristic-free approach. J. Pure Appl. Algebra, 151(1):43-50, 2000. 6

[Lyu06] Gennady Lyubeznik. On some local cohomology invariants of local rings. Math. Z., 254(3):627640, 2006. 1

[Mil00] Ezra Miller. The Alexander duality functors and local duality with monomial support. $J$. Algebra, 231(1):180-234, 2000. 3

[Mus00] Mircea Mustaţă. Local cohomology at monomial ideals. J. Symbolic Comput., 29(4-5):709720, 2000. Symbolic computation in algebra, analysis, and geometry (Berkeley, CA, 1998). 3, 7

[NBW13] Luis Núñez-Betancourt and Emily E. Witt. Lyubeznik numbers in mixed characteristic. Math. Res. Lett., 20(6):1125-1143, 2013. 1

[NBW14] Luis Núñez-Betancourt and Emily E. Witt. Generalized Lyubeznik numbers. Nagoya Math. J., 215:168-202, 2014. 1, 6

[NBWZ14] Luis Núñez-Betancourt, Emily E. Witt, and Wenliang Zhang. A survey on the Lyubeznik numbers. Preprint, arXiv:1210.2026 [math.AC], 2014. 2

[Pee11] Irena Peeva. Graded syzygies, volume 14 of Algebra and Applications. Springer-Verlag London, Ltd., London, 2011. 3, 5, 15

[PS73] C. Peskine and L. Szpiro. Dimension projective finie et cohomologie locale. Applications à la démonstration de conjectures de M. Auslander, H. Bass et A. Grothendieck. Inst. Hautes Études Sci. Publ. Math., (42):47-119, 1973. 5

[Put13] Tony J. Puthenpurakal. On injective resolutions of local cohomology modules. Preprint, arXiv:1312.5852 [math.AC], 2013. 1, 8

[Sba01] Enrico Sbarra. Upper bounds for local cohomology for rings with given Hilbert function. Comm. Algebra, 29(12):5383-5409, 2001. 5

[Swi14] Nicholas Switala. Lyubeznik numbers for nonsingular projective varieties. Preprint, arXiv:1403.5776 [math.AC], 2014. 1

[Ter99] N. Terai. Local cohomology with respect to monomial ideals. Preprint, 1999. 3

[Yan00] Kohji Yanagawa. Alexander duality for Stanley-Reisner rings and squarefree $\mathbf{N}^{n}$-graded modules. J. Algebra, 225(2):630-645, 2000. 3

[Yan01] Kohji Yanagawa. Bass numbers of local cohomology modules with supports in monomial ideals. Math. Proc. Cambridge Philos. Soc., 131(1):45-60, 2001. 3, 4, 6, 11, 14, 15

[Yan04] Kohji Yanagawa. Derived category of squarefree modules and local cohomology with monomial ideal support. J. Math. Soc. Japan, 56(1):289-308, 2004. 9

[Yan12] Kohji Yanagawa. Sliding functor and polarization functor for multigraded modules. Comm. Algebra, 40(3):1151-1166, 2012. 3, 5, 13

[Zha07] Wenliang Zhang. On the highest Lyubeznik number of a local ring. Compos. Math., 143(1):8288, 2007. 1, 3

[Zha11] Wenliang Zhang. Lyubeznik numbers of projective schemes. Adv. Math., 228(1):575-616, 2011. 1

Department of Mathematics, University of Virginia, Charlottesville, VA 229044137

Email address: ab4cb@virginia.edu

Department of Mathematics, University of Virginia, Charlottesville, VA 229044135

Email address: 1cn8m@virginia.edu

Department of Mathematics, Kansai university, Suita 564-8680, Japan

Email address: yanagawa@ipcku.kansai-u.ac.jp 